\title{
An Investigation on the Sensitivity of Wavelet Packet Modulation to Time Synchronization Error
}

\author{
M. K. Lakshmanan • D. Karamehmedović • \\ H. Nikookar
}

Published online: 8 September 2010

(c) The Author(s) 2010. This article is published with open access at Springerlink.com

\begin{abstract}
Wavelet Packet based Multi-Carrier Modulation (WPMCM) offers an alternative to the well-established OFDM as an efficient multicarrier modulation technique. It has the advantage of being a generic transmission scheme whose actual characteristics can be widely customized to fulfill several requirements and constraints of advanced communication systems. In the last decades wavelets have been favorably applied in signal and image processing fields but only recently have they attracted the attention of the telecommunication community. A lot of research questions remain to be addressed before the novel WPMCM can be used in practice. One of the major concerns is the performance of WPMCM transceivers under time synchronization errors. This is important because WPMCM symbols overlap in the time domain. In this paper, we analyze the interference in WPMCM transmission caused by the time synchronization errors. The expressions for Inter Carrier Interference (ICI) and Inter Symbol Interference (ISI) in WPMCM transmission are derived and then through simulation studies, the ability of wavelet-based systems to cope with time synchronization errors is evaluated.
\end{abstract}

Keywords Wavelet packets · Multi-carrier modulation · Time offset errors · OFDM

\section{Introduction}

Multicarrier modulation (MCM) techniques have gained popularity in the last few decades due to higher bandwidth requirements, increased use of wireless systems in time-dispersive

M. K. Lakshmanan ( $\varangle) \cdot$ D. Karamehmedović · H. Nikookar International Research Center for Telecommunications and Radar (IRCTR), Department of Electrical Engineering, Mathematics and Computer Science, Delft University of Technology, Mekelweg 4, 2628 CD Delft, The Netherlands e-mail: m.k.lakshmanan@tudelft.nl

H.Nikookar

e-mail: h.nikookar@tudelft.nl 
environments (e.g. home, office, etc.) and lower cost of digital signal processing components. It is a spectral efficient modulation scheme which divides the incoming high rate data among multiple carriers modulated at lower rates. By simultaneously transmitting $N$ data symbols into $N$ carriers the symbol rate is reduced to the one $N$ th of the original symbol rate, and therefore the symbol duration is increased by $N$ times. This leads to a transmission system which is robust against channel dispersions/fading, impulse noise and multipath interference.

Traditionally, the Fast Fourier Transform (FFT) based Orthogonal Frequency Division Multiplexing (OFDM) has been recognized as the most cost-effective and bandwidth efficient realization of multicarrier transceivers. In the last 15 years OFDM has been widely adopted and standardized across the world. Few of the applications and standards which use OFDM are Digital Audio Broadcasting (DAB), Digital Video Broadcasting (DVB), WiFi (IEEE $802.11 \mathrm{a} / \mathrm{g} / \mathrm{j} / \mathrm{n}$ ), World Wide Interoperability for Microwave Access (WiMAX - IEEE 802.16), Ultra Wide Band wireless Personal Area Network (UWB Wireless PAN-IEEE 802.15.3a) and Mobile Broadband Wireless Access (MBWA-IEEE 802.20).

Recently, wavelet transformation has emerged as a strong candidate for digital modulation $[1,2]$. Wavelet Packet based Multi-Carrier Modulation (WPMCM) ${ }^{1}$ is a novel multicarrier modulation technique and a promising alternative to the well established OFDM. WPMCM was first proposed in [3] where the theoretical foundations were laid out for this novel orthogonal MCM technique and its use as an alternative to OFDM was propounded. The greatest motivation for pursuing WPMCM systems lies in the freedom they provide to communication systems designers. Unlike the Fourier bases which are static sines/cosines, WPMCM uses wavelets which offer flexibility and adaptation that can be tailored to satisfy an engineering demand. By tailoring the design specifications, a wavelet based system that best suits a wireless engineering requirement could be conceived.

The fundamental theories of OFDM and WPMCM have many similarities in their way of functioning and performance, particularly, with respect to the use of orthogonal subcarriers which overlap in the frequency domain and yet do not interfere with one another. However, the radio front-end induced impairments such as frequency offset and/or phase noise can cause the subcarriers to lose their mutual orthogonality and to begin interfering with one another. The rise of interference level due to loss of orthogonality among subcarriers is a major issue in multicarrier transmission. Therefore, the multicarrier systems with overlapping subcarriers are much more sensitive to the frequency offset and phase noise when compared to single carrier systems. This disadvantage of multicarrier systems sets high demands on the quality of the analog radio parts, like oscillator stability. In case of OFDM, the effects of frequency offset and phase noise are well documented in the literature [4-10] and a number of synchronization techniques exist to estimate and reduce the frequency offset and phase noise effects [11-17].

Besides errors in the form of frequency and phase misalignment, the multicarrier transceiver can also suffer from a loss of synchronization between the transmitter and receiver. Time synchronization error occurs when the start of the multicarrier symbol is incorrectly detected, selecting parts of the adjacent symbol while discarding some samples at the beginning or end of the useful symbol. Due to time synchronization errors, infarctions such as the Inter Symbol Interference (ISI) and Inter Carrier Interference (ICI) arise. The use of guard intervals, like cyclic prefix in OFDM, can significantly improve the system performance in case of timing errors. However, the use of guard interval is not feasible solution for WPMCM systems because of time-overlapping nature of wavelet packet transform. Alike the study on

1 This paper was presented in part at the 11th International Symposium on Wireless Personal Multimedia Communication (WPMC) 2008, Lapland, Finland and the 19th Personal, Indoor and Mobile Radio Communications (PIMRC), September 2008, Cannes, France. 
the impact of frequency offset and phase noise on the performance of the system, far more documentation exists on the operation of OFDM under timing errors than for WPMCM. The sensitivity of the OFDM to the time synchronization error is reported in [18-20] and various techniques for OFDM symbol synchronization can be found in [16-23].

In this article we will address the time synchronization errors for the WPMCM transceiver, and compare the performance of WPMCM under these errors to OFDM. Furthermore, the performance of WPMCM transceivers employing some standard wavelets will be numerically evaluated under different conditions.

The rest of the paper is organized as follows. The system blocks of OFDM and WPMCM transceivers are outlined in Sect.2. Time offset error is discussed in Sect.3. In Sect.4 the modulation technique used in the study is explained. In Sect.5 the results obtained by computer simulation for time offset errors are shown. Finally, the paper is concluded in Sect.4.

\section{Multicarrier Transceivers}

\section{$2.1 \mathrm{OFDM}$}

OFDM transmission system can be efficiently implemented using Inverse Fast Fourier Transform (IFFT) at the transmitter side and FFT at the receiver side. The transmitter and receiver blocks of an OFDM communication system are illustrated in Figs. 1 and 2, respectively.

Each OFDM symbol contains $N$ subcarriers, a number that is determined by the size of FFT. If an OFDM system has a symbol period of $T$ and uses $N$ subcarriers with intercarrier spacing $\Delta f=1 / T$, the output of the transmitter in one symbol period can be expressed as:

$$
S(n)=\sum_{k=0}^{N-1} a_{k} e^{j 2 \pi \frac{k n}{N}}, \quad 0 \leq n \leq N-1
$$

In (1) $a_{k}$ represents mapped complex data symbols which are obtained from binary input stream using one of the standard multilevel modulation techniques, such as $M$-QAM or $M$-PSK.

If we assume ideal channel and perfect synchronization between OFDM transmitter and receiver, the received sequence $R(n)$ is identical to the transmitted signal, i.e. $R(n)=S(n)$. Under these conditions the demodulated data after FFT for the $k^{\prime}$ th subcarrier can be expressed as

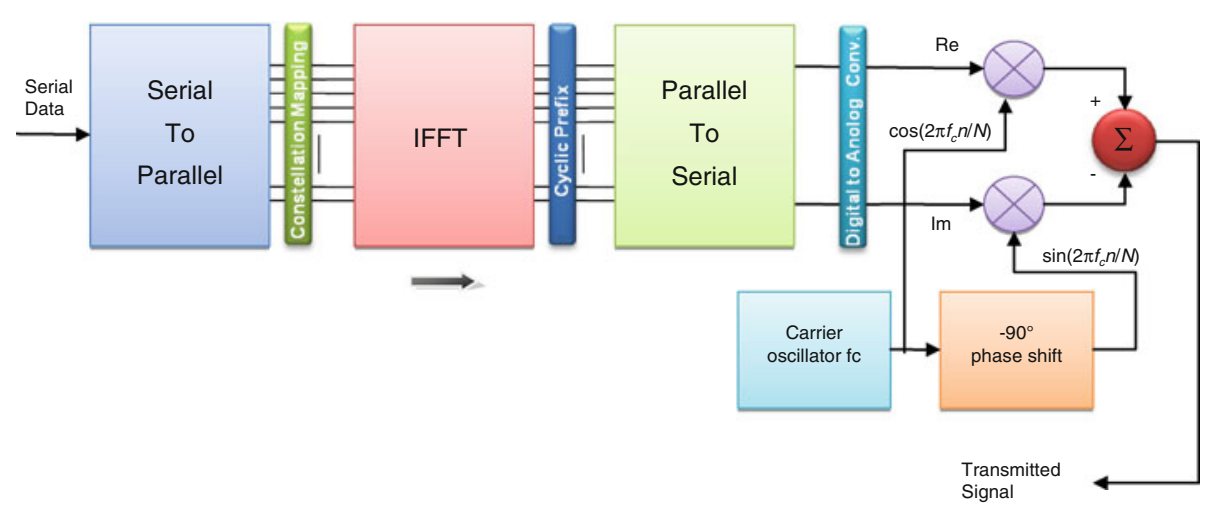

Fig. 1 OFDM transmitter 


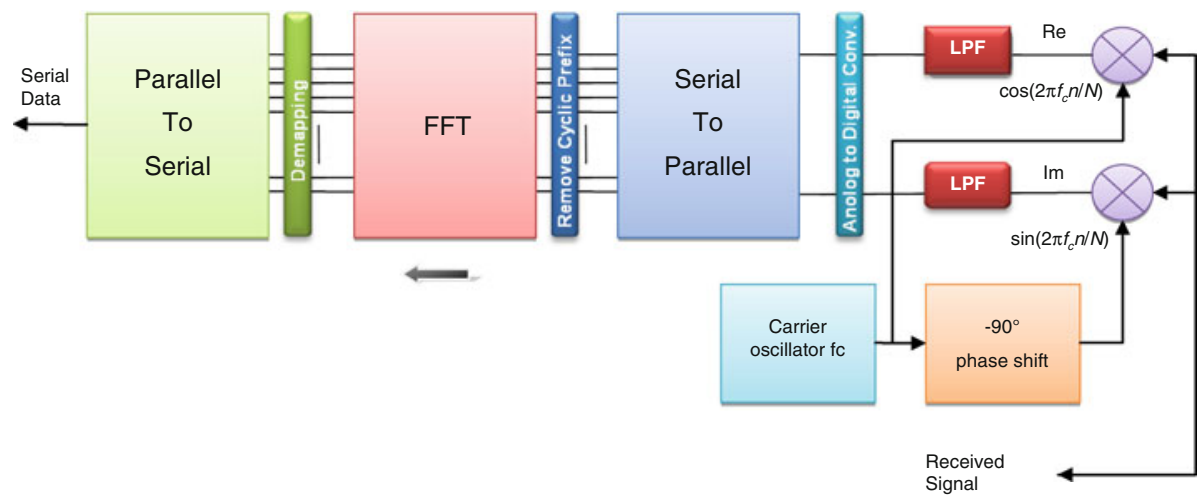

Fig. 2 OFDM receiver

$$
\begin{aligned}
\hat{a}_{k^{\prime}} & =\frac{1}{N} \sum_{n=0}^{N-1} R(n) e^{-j 2 \pi \frac{k^{\prime} n}{N}}=\frac{1}{N} \sum_{n=0}^{N-1} \sum_{k=0}^{N-1} a_{k} e^{j 2 \pi \frac{k n}{N}} e^{-j 2 \pi \frac{k^{\prime} n}{N}} \\
& =\sum_{k=0}^{N-1} a_{k}\left(\frac{1}{N} \sum_{n=0}^{N-1} e^{j 2 \pi \frac{n\left(k-k^{\prime}\right)}{N}}\right)=\sum_{k=0}^{N-1} a_{k} \delta\left(k-k^{\prime}\right)=a_{k^{\prime}}
\end{aligned}
$$

In (2) $\delta$ denotes the Kronecker delta function.

\subsection{WPMCM}

WPMCM is a multiplexing method that makes use of orthogonal wavelet packets waveforms to combine a collection of parallel signals into a single composite signal. Fundamentally, OFDM and WPMCM have many similarities as both use orthogonal waveforms as subcarriers and they achieve high spectral efficiency by allowing their subcarriers' spectra to overlap one another. The main differences between OFDM and WPMCM lie in the shape of the subcarriers and in way they are generated. One important property of wavelet based transformation is that the waveforms used in general are longer than the transform duration of one symbol. This causes WPMCM symbols to overlap in time domain. Thanks to the fulfillment of double shift orthogonality the overlap of the symbols does not lead to ISI. Longer waveforms allow better frequency localization of WPMCM's subcarriers while in OFDM the rectangular shape of DFT window generates large side lobes. Figure 3a,b illustrates the spectra of 8 adjacent subcarriers for WPMCM and OFDM transceiver, respectively.

The other deleterious consequence of time overlap is the inability to use guard intervals in wavelet based systems. Although adding guard intervals severely decrease spectral efficiency in OFDM, they are effective and low complexity method to cope with dispersive channels and time offset.

WPMCM employs the Inverse Discrete Wavelet Packet Transform (IDWPT) at the transmitter side and Discrete Wavelet Packet Transform (DWPT) at the receiver side, analogous to the IFFT and FFT used by the OFDM transceivers. The IDWPT is implemented by wavelet packet synthesis filter bank which combines different parallel streams into a single signal. This composite signal is afterwards decomposed at the receiver using wavelet packets analysis filter bank or so called DWPT. 

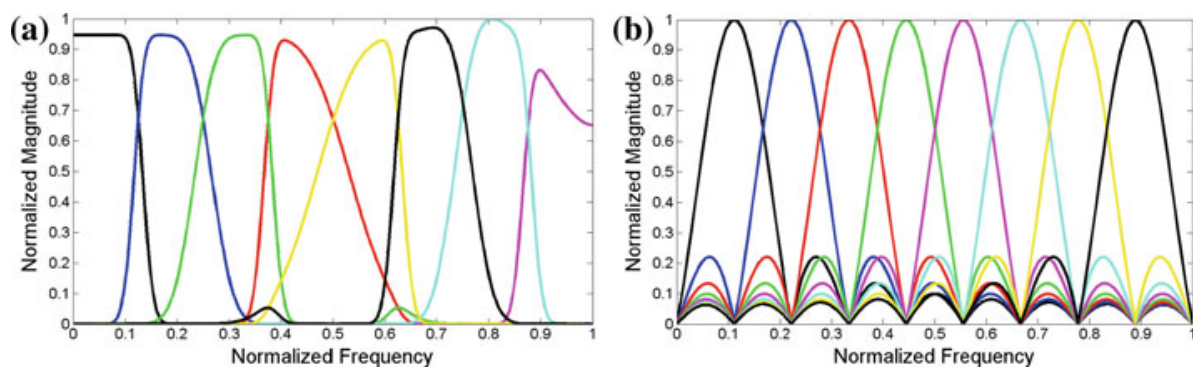

Fig. 3 Spectrum of 8 WPMCM subcarriers (a) (Daubechies wavelet with 20 coefficients); (b) Spectrum of 8 OFDM subcarriers

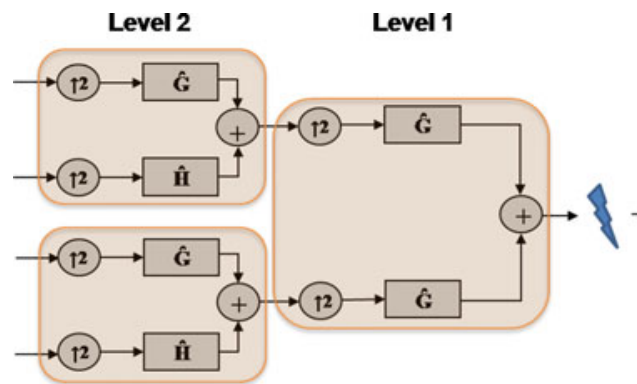

\section{Synthesis Filter Bank - Transmitter Side}

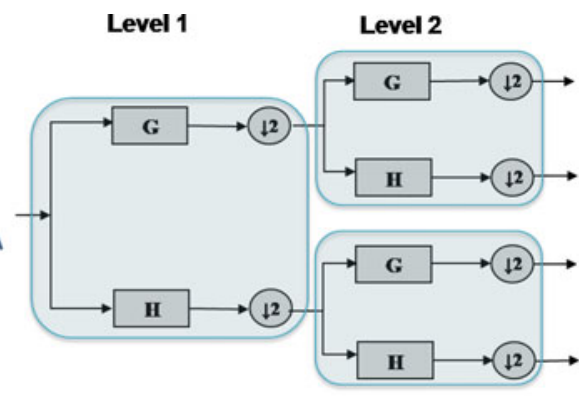

Analysis Filter Bank Receiver Side

Fig. 4 Wavelet packed based Transmultiplexer Left: synthesis tree, Right: analysis tree

In Fig. 4a 4-channel wavelet packed based transmultiplexer is illustrated. The upsampling and downsampling operations by a factor 2 are represented by $\uparrow 2$ and $\downarrow 2$, respectively, while filter $G$ stands for high-pass wavelet filter and filter $H$ is corresponding low-pass scaling filter.

The wavelet packet analysis and synthesis trees can be efficiently constructed by iteration of corresponding 2-channel filter bank. Therefore, we can calculate the wavelet packet waveforms in a recursive manner using the following algorithm

$$
\begin{aligned}
& \xi_{l+1}^{2 p}(t)=\sqrt{2} \sum_{n} h(n) \xi_{l}^{p}\left(t-2^{l} n\right) \\
& \xi_{l+1}^{2 p+1}(t)=\sqrt{2} \sum_{n} g(n) \xi_{l}^{p}\left(t-2^{l} n\right)
\end{aligned}
$$

In (3) the wavelet packet waveforms are denoted by $\xi$ while superscript $p$ can be seen as subcarrier index at a given tree depth (level l). The scaling and wavelet filter coefficients in (3) are represented by $h(n)$ and $g(n)$, respectively.

Because WPMCM transceivers are realized by an iterative method we can easily change the number of subcarriers and their bandwidth. By performing an additional iteration of 2-channel filter bank at all outputs the subcarriers number is doubled or more generally the number of subcarriers is given by

$$
N=2^{l}
$$


The subcarriers in WPMCM transceivers are completely determined by filters $H$ and $G$ and therefore by applying different set of filters we get different subcarriers which in turn lead to different transmission system characteristics. References [3,24-28] have shown that by merely altering the filter coefficients the WPMCM transceivers can be adjusted to operate with different bandwidth efficiency, frequency concentration of subcarriers, sensitivity to synchronization errors, PAPR, etc.

Filters in WPMCM cannot be arbitrarily chosen and not all scaling and wavelet filters will fit the requirements for a communication system. First of all, the filters need to have a Finite Impulse Response (FIR) because they allow wavelet packet transformation to be implemented using fast recursive algorithm. Furthermore, for perfect reconstruction of the transmitted signal the subcarriers have to be orthogonal. These can only be generated by filters that fulfill the double shift orthogonality constraint [29]. The WPMCM's waveforms are mutually orthogonal if they satisfy the following condition:

$$
\left\langle\xi_{l}^{p}(t), \xi_{l}^{i}(t)\right\rangle=\sum_{k} \xi_{l}^{p}(t) \xi_{l}^{i}(t)=\delta(p-i)
$$

In (5) the angle brackets stand for the inner product between two waveforms.

The WPMCM signal consists of compartmentalized frames which in turn contain multicarrier symbols obtained by summing modulated subcarriers. Since the filters used to derive the waveforms are longer than one multicarrier symbol duration, the whole WPMCM frame has to be processed at once. The WPMCM signal in the discrete time domain can be expressed as:

$$
S(n)=\sum_{u} \sum_{k=0}^{N-1} a_{u, k} \xi_{\log _{2}(N)}^{k}(n-u N)
$$

In (6) $k$ denotes the subcarrier index and $u$ denotes the WPMCM symbol index. The constellation symbol modulating $k$ th subcarrier in $u$ th WPMCM symbol is represented by $a_{u, k}$.

The receiver demodulates the data by employing time reversed version of waveforms used by the transmitter. If we assume that the WPMCM transmitter and receiver are perfectly synchronized and that the channel is ideal, the detected data at the receiver output can be expressed as:

$$
\begin{aligned}
\hat{a}_{u^{\prime}, k^{\prime}} & =\sum_{n} R(n) \xi_{\log _{2}(N)}^{k^{\prime}}\left(u^{\prime} N-n\right)=\sum_{n} \sum_{u} \sum_{k=0}^{N-1} a_{u, k} \xi_{\log _{2}(N)}^{k}(n-u N) \xi_{\log _{2}(N)}^{k^{\prime}}\left(u^{\prime} N-n\right) \\
& =\sum_{u} \sum_{k=0}^{N-1} a_{u, k}\left(\sum_{n} \xi_{\log _{2}(N)}^{k}(n-u N) \xi_{\log _{2}(N)}^{k^{\prime}}\left(u^{\prime} N-n\right)\right) \\
& =\sum_{u} \sum_{k=0}^{N-1} a_{u, k} \delta\left(k-k^{\prime}\right)=a_{u^{\prime}, k^{\prime}}
\end{aligned}
$$

In Figs. 5 and 6 the transmitter and receiver blocks of a WPMCM communication system are illustrated. 


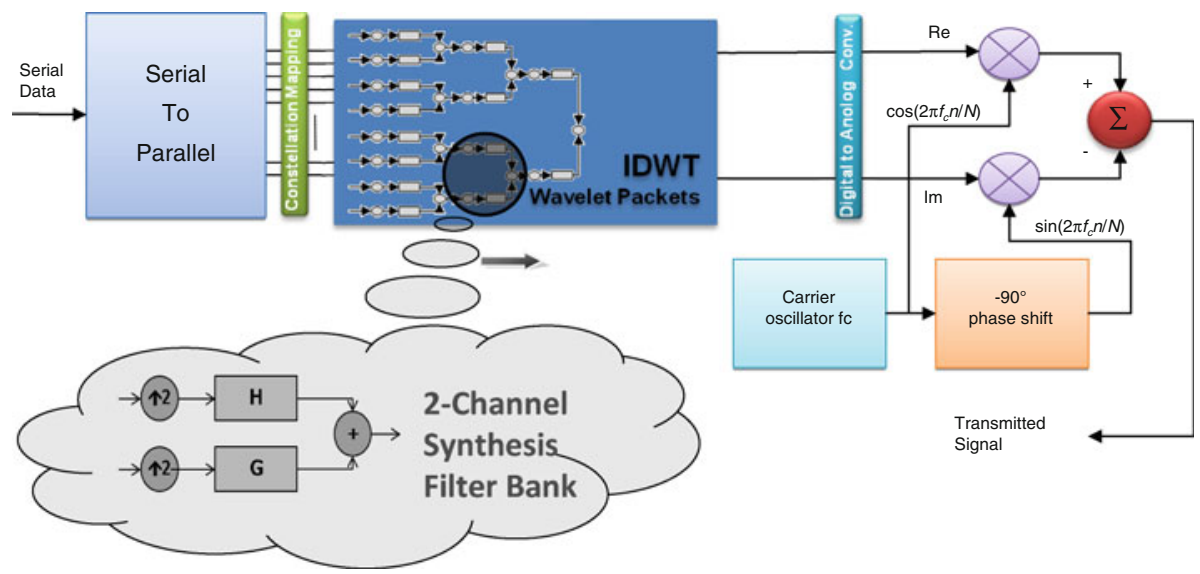

Fig. 5 WPMCM transmitter

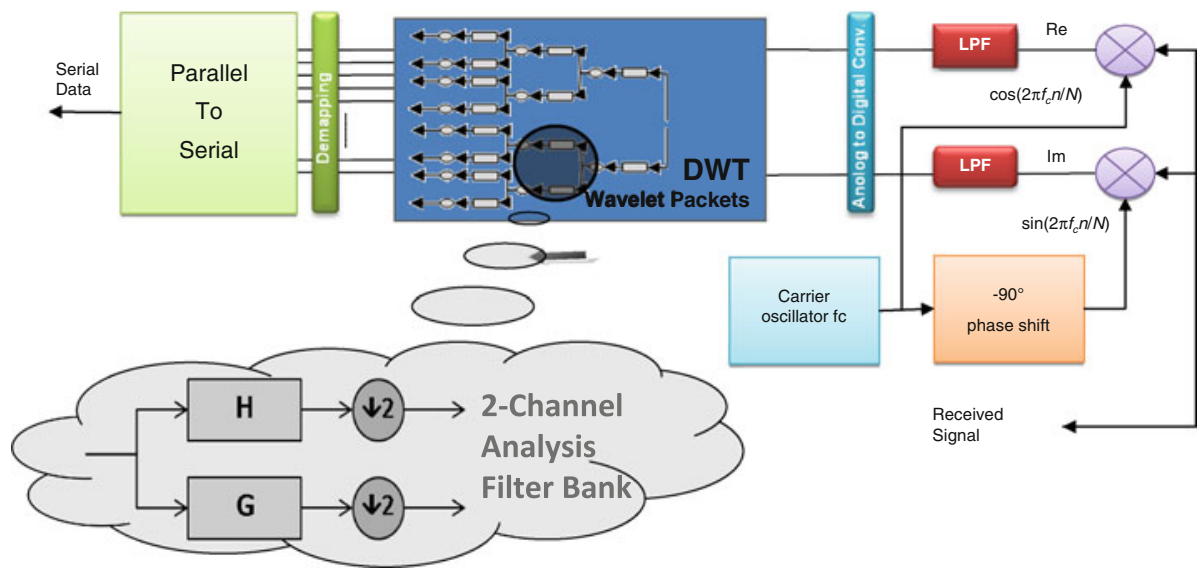

Fig. 6 WPMCM receiver

\section{Time Offset in Multicarrier Modulation}

One of the major concerns of a multicarrier system is their vulnerability to timing synchronization errors, which occur when multi-carrier symbols are not perfectly aligned at the receiver. Because of the time offset, samples outside a WPMCM or OFDM symbol get erroneously selected, while useful samples at the beginning or at the end of that particular symbol get discarded.

The time synchronization error is modeled by shifting the received data samples by a time offset value $t_{\varepsilon}$ to the left or right, depending on the sign of the $t_{\varepsilon}$. Time offset degrades the performances of multicarrier transceivers by introducing inter-symbol interference (ISI) and inter-carrier interference (ICI). WPMCM and OFDM share many similarities as both are orthogonal multicarrier systems but in case of timing error there is a major difference that causes different behaviors for each transmission scheme.

The actual length of the WPMCM symbols is defined by the wavelet used and in general it is significantly longer than the OFDM symbol. This excessive length of WPMCM symbols 


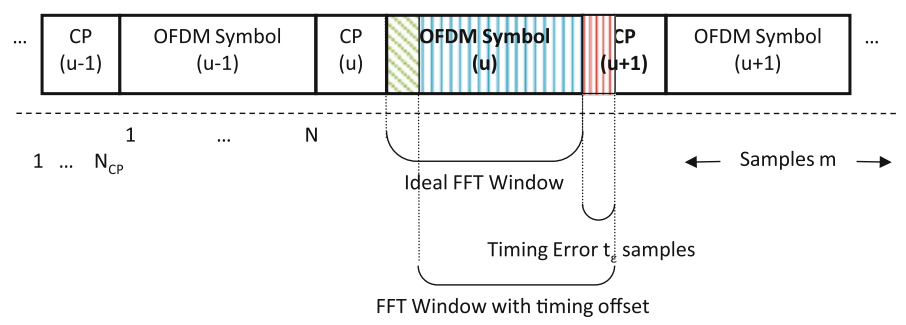

Fig. 7 Timing offset away from cyclic prefix (to the right)

does not cause frame size to grow by allowing symbols to overlap one another. In case of time offset this overlap of the symbols in WPMCM causes each symbol to interfere with several other symbols while in OFDM each symbol only interferes with adjacent symbols.

The second important difference between the two transmission schemas is the use of the guard interval between the symbols. OFDM uses cyclic prefix that significantly improves its performance when time errors occur, assuming that time offset is not exceeding the size of cyclic prefix and that the direction of time shift is towards the cyclic prefix. The WPMCM, on the other hand, cannot benefit from such guard interval since the WPMCM symbols overlap over one another.

\subsection{Time Offset in OFDM}

Adding a Cyclic prefix is an effective and low complexity method to cope with dispersive channels and time synchronization errors in OFDM transceivers. Two distinct situations can occur under time synchronization errors, depending on the direction of the time offset.

- Time synchronization error away from own cyclic prefix (to the right).

- Time synchronization error towards own cyclic prefix (to the left).

\subsubsection{Time Offset Away From the Cyclic Prefix}

Figure 7 illustrates 3 OFDM symbols $(u-1, u, u+1)$ where FFT window is misaligned to the right, i.e. away from the cyclic prefix. Each OFDM symbol consists of $N$ data samples and an extension of $N_{C P}$ samples representing cyclic prefix. The FFT window in the situation illustrated will contain $N-t_{\varepsilon}$ data samples $\left(\left(t_{\varepsilon}+1\right),\left(t_{\varepsilon}+2\right), \ldots, N\right)$ of the considered $u$ th OFDM symbol, missing first $t_{\varepsilon}$ time offset samples. Instead $t_{\varepsilon}$ samples $\left(1,2, \ldots, t_{\varepsilon}\right)$ of the next $(u+1)$ th symbol will be erroneously selected.

An OFDM system that is affected by timing error and where samples of neighboring symbol are wrongly selected experience severe degradation of the performance. The demodulated OFDM signal after FFT can be written as:

$$
\begin{aligned}
\hat{a}_{u^{\prime}, k^{\prime}}= & \underbrace{\frac{N-t_{\varepsilon}}{N} a_{u^{\prime}, k^{\prime}} e^{j 2 \pi \frac{k^{\prime}}{N} t_{\varepsilon}}}_{\text {Useful Signal }}+\underbrace{\frac{1}{N} \sum_{n=0}^{N-1-t_{\varepsilon}} \sum_{k=0 ; k \neq k^{\prime}}^{N-1} a_{u^{\prime}, k} e^{j 2 \pi \frac{k\left(n+t_{\varepsilon}\right)}{N}} e^{-j 2 \pi \frac{k^{\prime} n}{N}}}_{\text {ICI }} \\
+ & \underbrace{\frac{1}{N} \sum_{n=N-t_{\varepsilon}}^{N-1} \sum_{k=0}^{N-1} a_{u+1, k} e^{j 2 \pi \frac{k\left(n-N+t_{\varepsilon}\right)}{N}} e^{-j 2 \pi \frac{k^{\prime} n}{N}}}_{\text {ISI }}
\end{aligned}
$$




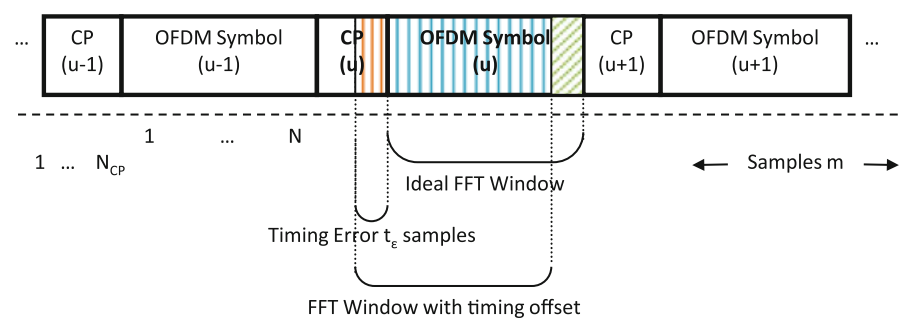

Fig. 8 Timing offset towards cyclic prefix (to the left)

The first component of Eq. (8) represents useful signal which is attenuated and phase shifted by a term proportional to subcarrier index $k^{\prime}$. The second component of (8) gives ICI and the third component stands for ISI with next symbol.

\subsubsection{Time Offset Towards the Cyclic Prefix}

The other situation occurs when we have time offset towards the symbols own cyclic prefix, i.e. to the left. Figure 8 illustrates such a scenario. In this case FFT window consists of first $N-t_{\varepsilon}$ samples $\left(1,2, \ldots,\left(N-t_{\varepsilon}\right)\right)$ of the considered $u$ th OFDM symbol and the last $t_{\varepsilon}$ samples of the own cyclic prefix. We assume for the convenience that $t_{\varepsilon}<N_{C P}$.

The demodulated OFDM signal affected by time offset in the direction of symbol's own cyclic prefix is given in Eq. (9), for case when $t_{\varepsilon}<N_{C P}$.

$$
\hat{a}_{u^{\prime}, k^{\prime}}=a_{u^{\prime}, k^{\prime}} e^{-j 2 \pi \frac{k^{\prime} t_{\varepsilon}}{N}}
$$

Thanks to the cyclic prefix the orthogonality is preserved and ISI and ICI terms have disappeared. The timing error towards the cyclic prefix results therefore in pure phase shift.

\subsubsection{Time Offset in WPMCM}

The WPMCM transceivers do not apply guard intervals and therefore the direction of time offset is inconsequential. The demodulated signal under influence of time offset $t_{\varepsilon}$ can be written as:

$$
\begin{aligned}
\hat{a}_{u^{\prime}, k^{\prime}} & =\sum_{n} R(n) \xi_{\log _{2}(N)}^{k^{\prime}}\left(u^{\prime} N-n+t_{\varepsilon}\right) \\
& =\sum_{n} \sum_{u} \sum_{k=0}^{N-1} a_{u, k} \xi_{\log _{2}(N)}^{k}(n-u N) \xi_{\log _{2}(N)}^{k^{\prime}}\left(u^{\prime} N-n+t_{\varepsilon}\right) \\
& =\sum_{u} \sum_{k=0}^{N-1} a_{u, k}\left(\sum_{n} \xi_{\log _{2}(N)}^{k}(n-u N) \xi_{\log _{2}(N)}^{k^{\prime}}\left(u^{\prime} N-n+t_{\varepsilon}\right)\right)
\end{aligned}
$$

In order to shorten the derivation the following notation is defined:

$$
\Omega_{k, k^{\prime}}^{u, u^{\prime}}\left(t_{\varepsilon}\right)=\sum_{n} \xi_{\log _{2}(N)}^{k}(n-u N) \xi_{\log _{2}(N)}^{k^{\prime}}\left(u^{\prime} N-n+t_{\varepsilon}\right)
$$

Equation (11) represents the autocorrelation and the cross-correlation of the WPMCM waveforms. When $k=k^{\prime}$ the two subcarrier waveforms are time-reversed images of each other 
and Eq. (11) gives the autocorrelation sequence of the waveform $k$. In the other cases when $k \neq k^{\prime}$ the two waveforms correspond to different subcarriers and (11) stands for the crosscorrelation between waveforms $k$ and $k^{\prime}$.

Using Eqs. (10) and (11) we can now express the output of the WPMCM receiver for the $k$ th subcarrier and $u$ th WPMCM symbol as:

$$
\hat{a}_{u^{\prime}, k^{\prime}}=\underbrace{a_{u^{\prime}, k^{\prime}} \Omega_{k^{\prime} k^{\prime}}^{u^{\prime}, u^{\prime}}}_{\text {UsefulSignal }}+\underbrace{\sum_{u ; u \neq u^{\prime}} a_{u, k^{\prime}} \Omega_{k^{\prime}, k^{\prime}}^{u, u^{\prime}}}_{\text {ISI }}+\underbrace{\sum_{u} \sum_{k=0 ; k \neq k^{\prime}}^{N-1} a_{u, k} \Omega_{k, k^{\prime}}^{u, u^{\prime}}}_{\text {ICI }}
$$

In Eq. (12) the first term stands for attenuated useful signal. The second term gives the ISI due to symbols transmitted on the same subchannel and the third term denotes ICI measured over the whole frame.

The received constellation points of WPMCM under time synchronization errors do not experience linear phase rotation, opposed to OFDM where rotation of constellation points is proportional to subcarrier index. The WPMCM signal in presence of timing error will however be attenuated and it will suffer from ISI and ICI.

\section{Modulation Scheme}

The consequence of time-offset in OFDM, regardless of offset direction, is the introduction of phase shift. The phase shift is linearly proportional to the subcarrier index and the value of time offset. The rotation angle $\Phi_{t}(k)$ due to timing error is given by:

$$
\Phi_{t}(k)=\frac{2 \pi k t_{\varepsilon}}{N}
$$

Standard modulation techniques such as coherent (non-differential) Quadrature Phase Shift keying (QPSK) perform poorly under time synchronization error because the subcarriers with higher frequency indices experience greater phase shifts. And even a small timing offset (such as $t_{\varepsilon}=1$ ) results in a phase rotation of constellation symbols in order of $0<\Phi_{t}(k)<2 \pi$. The subcarrier with the highest frequency will therefore experience a phase shift of almost 360 degrees. If this phase shift is not corrected the majority of the detected data would be corrupted even without ICI or ISI.

The phase rotation due to timing error can be usually revised by pilot-symbol-aided channel estimation techniques or by use of differential constellation mapping. In this article we employ differential quadrature phase shift keying (DQPSK) in order to overcome this problem. In the DQPSK scheme the data is modulated on the basis of phase difference between two consecutive constellation symbols, thereby ensuring that adjacent subcarriers experience a phase shift which is independent of the carrier position. The phase rotation of constellation point $k$ is determined by applying a phase shift of $\Delta \Phi$ to the previous constellation symbol $k-1$. The difference in phase shift $\Delta \Phi$ is determined by the unmodulated data value assigned to subcarrier $k$, in case of DQPSK phase shift can be written as:

$$
\Delta \Phi_{b}=\frac{2(b-1) \pi}{4}, \quad b \in 1, \ldots, 4
$$

The phase difference between two consecutive DQPSK constellation symbols under timing errors becomes:

$$
\Delta \phi_{k, k-1}=e^{j\left(\Delta \Phi_{b}-\frac{2 \pi t_{\varepsilon}}{N}\right)}
$$


Fig. 9 OFDM received constellation points with timing error of $t_{\varepsilon}=1$ and an ideal channel; Left: QPSK, Right: DQPSK $\pi / 4$
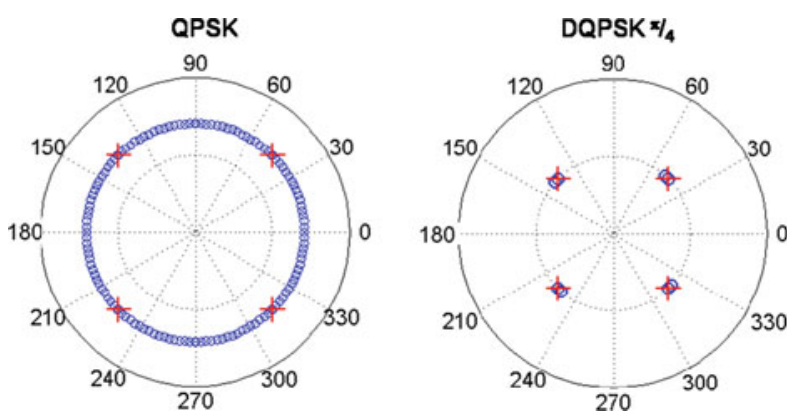

Using DQPSK modulation in presence of timing error therefore results in a phase shift that is depending on the value of the time offset but not anymore on the value of subcarrier index $k$. The rotation angle $\Phi_{t}(k)$ due to timing error becomes:

$$
\Phi_{t}(k)=\frac{2 \pi t_{\varepsilon}}{N}, \quad \text { differential PSK }
$$

Figure 9 illustrates the rotations of constellation points for received OFDM signal using QPSK and DQPSK modulation. We have assumed here an ideal channel and a time offset of $t_{\varepsilon}=1$ samples towards the cyclic prefix.

DQPSK modulation is a simple solution to overcome the problem of phase shift under time synchronization errors. However, DQPSK modulation requires about 2-3 dB higher SNR when compared to coherent QPSK to obtain the same BER performance in AWGN channel.

\section{Numerical Results for Time Offset}

The performances of WPMCM and OFDM in presence of timing synchronization errors are investigated by means of simulations. The time offset is modeled as a discrete uniform distribution between -2 and 2 samples, i.e. $t_{\varepsilon} \in[-2,-1,0,1,2]$.

First of all, DQPSK modulation scheme is used instead of QPSK modulation in order to prevent OFDM constellation points to experience to large phase shifts. Secondly, in OFDM we use cyclic prefix of 16 samples that is placed in front of OFDM symbols, in WPMCM we don't use any guard interval. Due to utilization of cyclic prefix the spectral efficiency of OFDM is decreased by $12.5 \%$ while spectral efficiency of WPMCM has remained unchanged.

The WPMCM signal consists of compartmentalized frames which in turn contain multicarrier symbols obtained by summing modulated subcarriers. Since the filters used to derive the waveforms are than one multicarrier symbol period, the whole WPMCM frame has to be processed at once.

Finally, we employ the oversampling in order to magnify the difference in performance between various systems and wavelets. An overview of simulation parameters is given in Table 1.

The nature and characteristics of the filters used in IDWPT and DWPT greatly influence the WPMCM carries derived. By changing the parameters of the filter design the carriercharacteristics can be customized to any requirement. WPMCM thus gives designers a lot of freedom that can be used to design case-characteristic systems. Having said that the wavelet bases cannot be arbitrarily chosen and instead have to satisfy a number of requirements. 
Table 1 Simulation setup time synchronization error

- Does not apply

Table 2 Wavelet specifications

- Does not apply

\begin{tabular}{lll}
\hline & WPMCM & OFDM \\
\hline Number of subcarriers & 128 & 128 \\
Number of multicarrier symbols per frame & 100 & 100 \\
Modulation & DQPSK & DQPSK \\
Channel & AWGN & AWGN \\
Oversampling Factor & 15 & 15 \\
Guard band & - & - \\
Guard interval & - & CP (length: 16) \\
Frequency offset & - & - \\
Phase noise & - & - \\
Time offset & $t_{\varepsilon}=2$ & $t_{\varepsilon}=2$ \\
\hline
\end{tabular}

\begin{tabular}{lll}
\hline Name & Length & Wavelet zero moment \\
\hline Daubechies & 40 & 20 \\
Symlets & 40 & 20 \\
Coiflet & 30 & 10 \\
Discrete meyer & 102 & - \\
Biorthog. $(2,2)$ & $(5,3)$ & 2 (dec.)
\end{tabular}

In general, the choices to make can be with regard to the system of representation (continuous or discrete), properties of the wavelets desired (orthogonality/biorthogonality, regularity/smoothness, frequency selectivity), the application in hand and the context of use [30].

In this effort we primarily consider the wavelet families Daubechies and its variants, Coiflet and Symlet. This is because these families provide orthonormal bases with compact support. Table 2 gives specifications of a few standard wavelets that were used during simulations.

Figure 10 illustrates some of the wavelet waveforms (in time and frequency domains) used in Table2 .

\subsection{Performance of WPMCM setup without Time offset}

To gauge the system operation we first check its performance under optimal conditions. Figure 11 shows the DQPSK constellation points for WPMCM setup using various wavelets in ideal channel conditions with no time offset. From the plot we may note that perfect estimates of the transmitted data can be obtained at the receiver when the transmitter and receiver ends are in time-unison.

A timing error results in a loss of time synchronization which causes a loss of orientation of incoming data at the receiver. As a result the time domain data entering the IDFT/IDWT block is incorrectly aligned whereby the samples of previous or next OFDM/WPMC symbol are selected while valid samples at the beginning or at the end of the symbol in consideration are discarded. We present the impact of time synchronization error in the sections $B$ and $C$. 

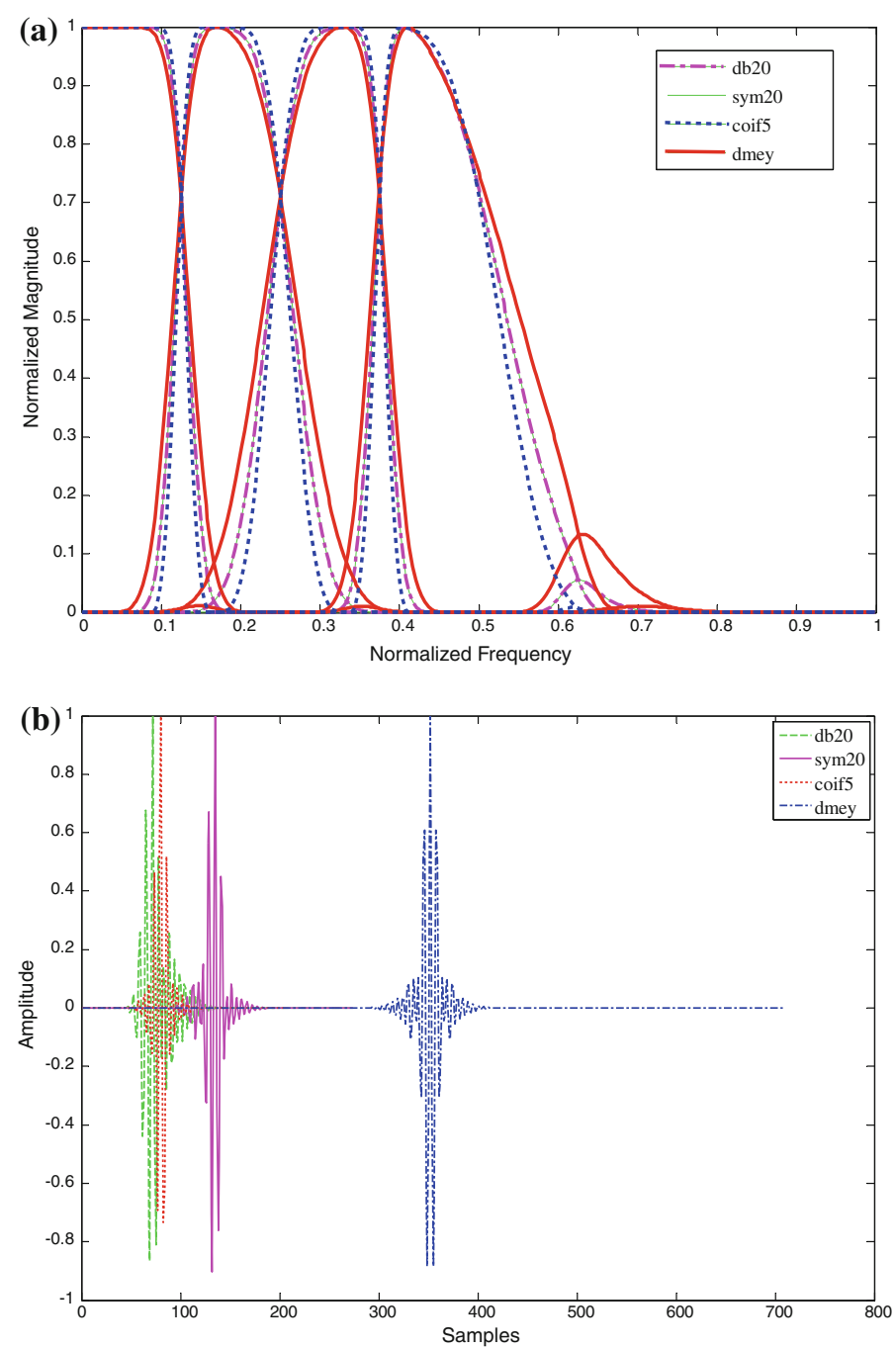

Fig. 10 Wavelet packet bases as MCM carriers (a) in time domain and (b) in frequency domain (only first 4 carriers shown). The wavelets considered are Daubechies-20, Symlet-20, Coiflet-5 and Discrete Meyer. The Daubechies-20 and Symlet-20 responses almost match and hence the responses of Symlet-20 are not visible

\subsection{Performance when Time offset is modeled as Discrete Uniform Distribution}

Figure 12 illustrates the bit error rates (BER) of OFDM and WPMCM transceivers over AWGN channel for uniformly distributed timing offset of $t_{\varepsilon}=2$ samples.

The OFDM system performs much better under time synchronization errors when compared to the WPMCM, partly due to exploitation of cyclic prefix and cancellation of phase rotation by DQPSK modulation scheme. The WPMCM cannot profit from these revisions and therefore show poor performance in presence of timing error. The biorthogonal wavelet has highest BER due to unfulfilled perfect reconstruction condition. 

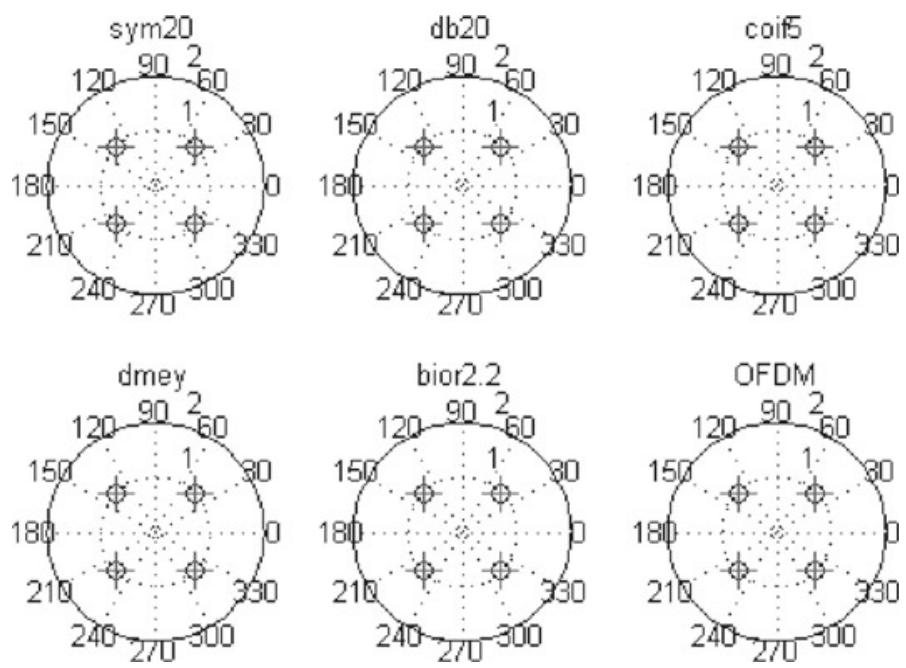

Fig. 11 DQPSK constellation points for WPMCM setup using various wavelets in ideal channel conditions with no time offset

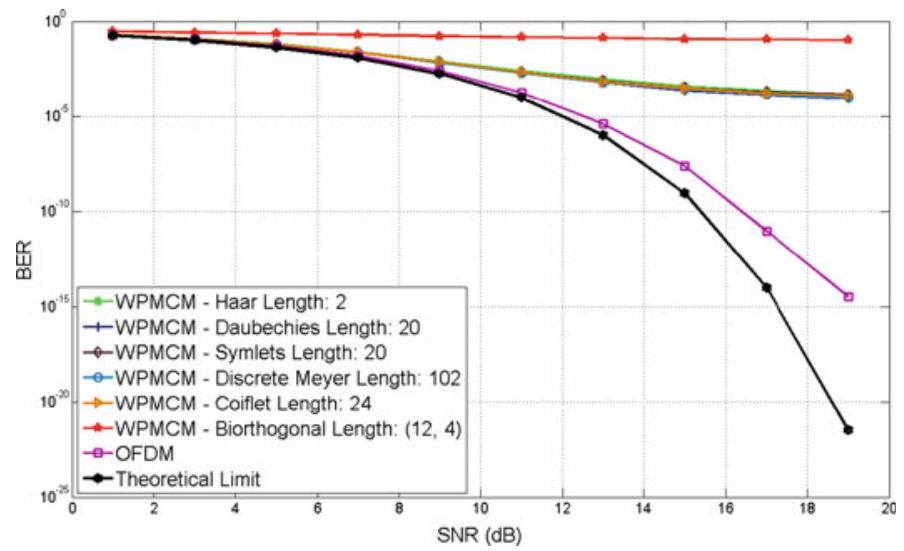

Fig. 12 BER for WPMCM with different wavelets and OFDM under time synchronization errors $\left(t_{\varepsilon}=2\right)$

In Fig. 13 the BER is shown for different values of time offset varying from -15 to 12 samples. The time offset in this simulation is modeled as one-sided uniform distribution in order to highlight the importance of cyclic prefix for OFDM. The value of time offset is given by $t_{\varepsilon} \in\left[0, \ldots, t_{\varepsilon}\right]$ for timing error to the right and by $t_{\varepsilon} \in\left[-t_{\varepsilon}, \ldots, 0\right]$ for the timing error to the left. During this simulation we kept SNR constant at $10 \mathrm{~dB}$.

The direction of time offset is inconsequential for WPMCM systems as can be seen at Fig.13. The BER curves of WPMCM are almost perfect mirror images with respect to the origin. This does not hold for OFDM, since we can see clearly that the negative timing offset (towards the own cyclic prefix) result in much lower BER when compared to the positive timing offsets (away from the own cyclic prefix). Due to use of cyclic prefix the misalignment of FFT window between the boundaries of extended symbols does not cause interference. However, when time offset exceeds the length of cyclic prefix the ICI and ISI terms reappear. 


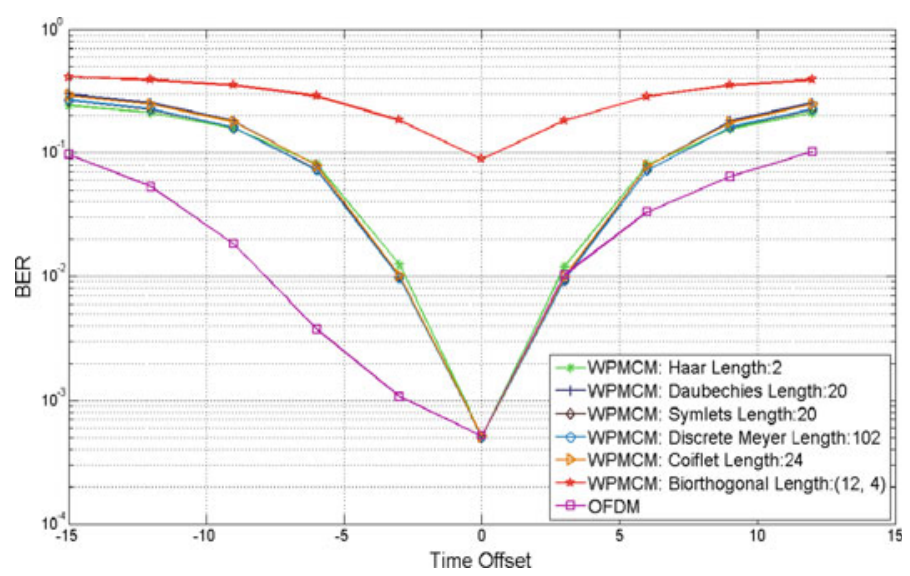

Fig. 13 BER versus time offset (in samples) for WPMCM and OFDM in AWGN channel $(\mathrm{SNR}=10 \mathrm{~dB})$
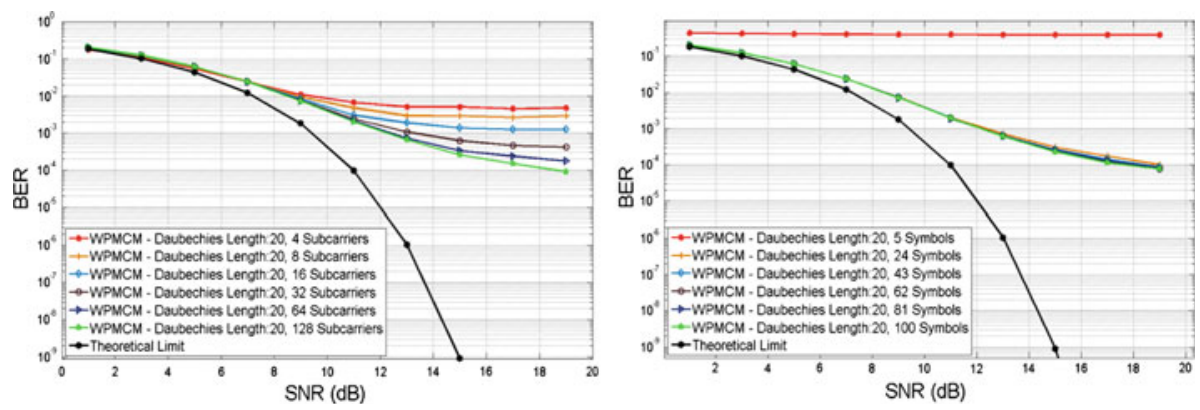

Fig. 14 BER for WPMCM with timing error; Left: Different number of subcarriers, Right: Different number of symbols

\subsection{Influence of number of subcarriers on the system performance}

Figure 14 shows the performance of the WPMCM in presence of time synchronization error when the number of subcarriers and symbols in the frame is altered.

The left side of Fig. 14 reveals that under timing error the performance of WPMCM depends on the number of subcarriers. For increasing number of WPMCM subcarriers the BER significantly decreases. We recall from the first chapter that the symbol duration of multicarrier system is proportional to the number of subcarrier used. Therefore, larger number of subcarriers means longer symbol duration and hence smaller relative time offset with respect to multicarrier symbol length.

The simulation results performed for different number of WPMCM symbols haven't shown any essential connection between BER performance and the number of symbols, when we assume that the number of symbols in a frame is exceeding the filter's length. Figure 15 illustrates the influence of filter's length and number of zero wavelet moments in combination with timing error on the BER. Daubechies filter with 6 coefficients and 3 wavelet zero moments has slightly higher BER when compared to longer filters from the same family. However, when length of the filters is further increased the BER curves become closely spaced and therefore we can conclude that there is no significant relation between 


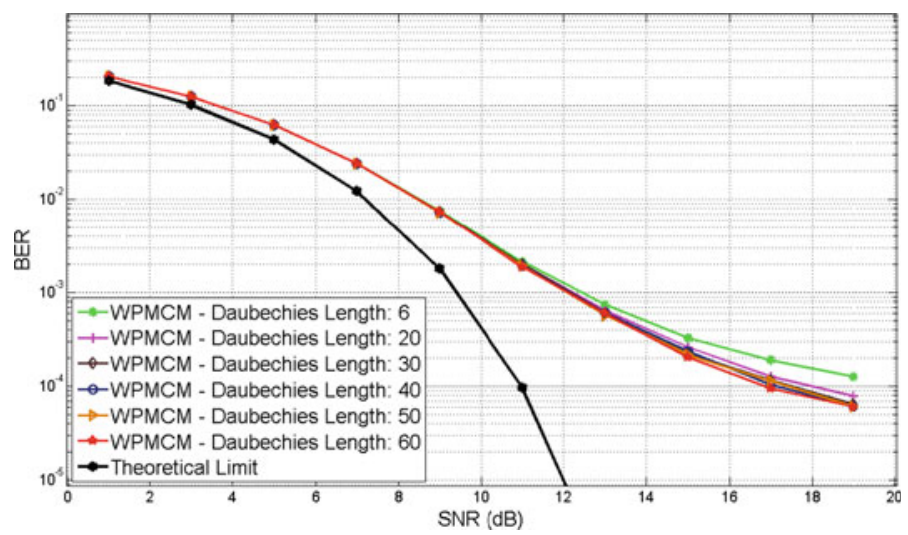

Fig. 15 BER for WPMCM using Daubechies wavelets of different lengths under influence of timing error

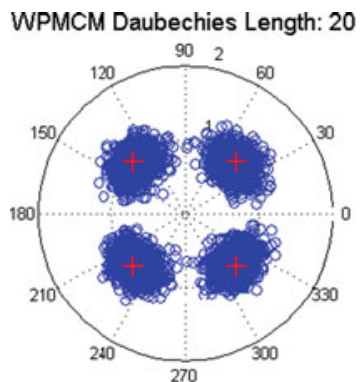

WPMCM Coiflet Length: 24

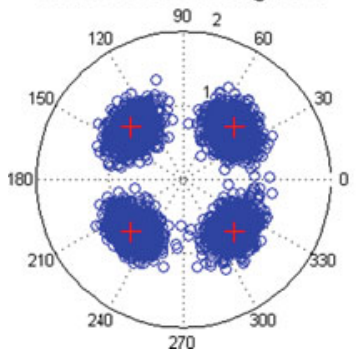

WPMCM Symlets Length: 20

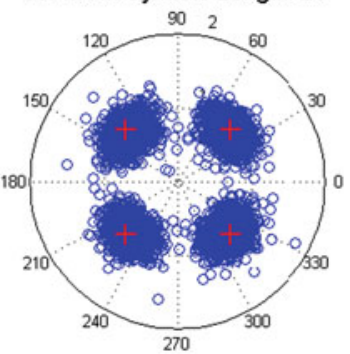

WPMCM Biorthogonal Length: $(12,4)$

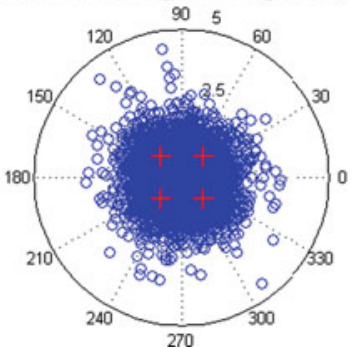

WPMCM Discrete Meyer Length: 102
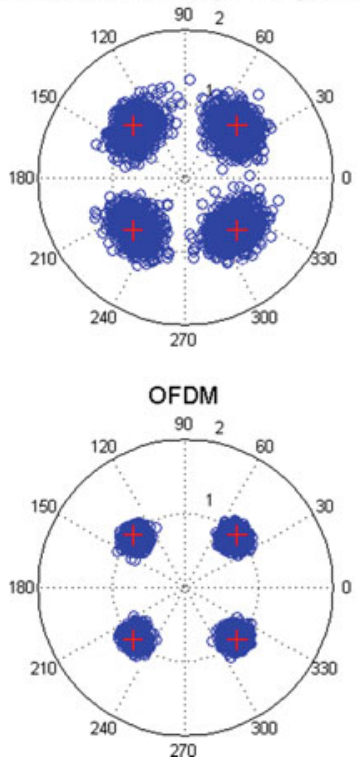

Fig. 16 Constellation points in the presence of timing error

the BER and filter's length in presence of timing errors. The effect of time synchronization error on the constellation points is depicted in the Fig. 16. In order to highlight the effect of time synchronization error we assumed for the moment an ideal channel without any noise.

The main consequence of the time offset is the scattering of the constellation points around reference positions due to interference. OFDM has more concentrated constellation points than any tested WPMCM system, which indicates that the signal to interference ratio (SIR) of OFDM is higher than SIR of WPMCM under timing errors.

Figure 17 illustrates the spreading of subcarrier energy due to time synchronization error. The timing error in OFDM results in ISI between successive symbols and ICI. If the cyclic prefix is used the ICI and ISI terms are cancelled for time offset towards the symbols own cyclic prefix, i.e. time offset to the left. This can be also seen in Fig.17 where energy of the 

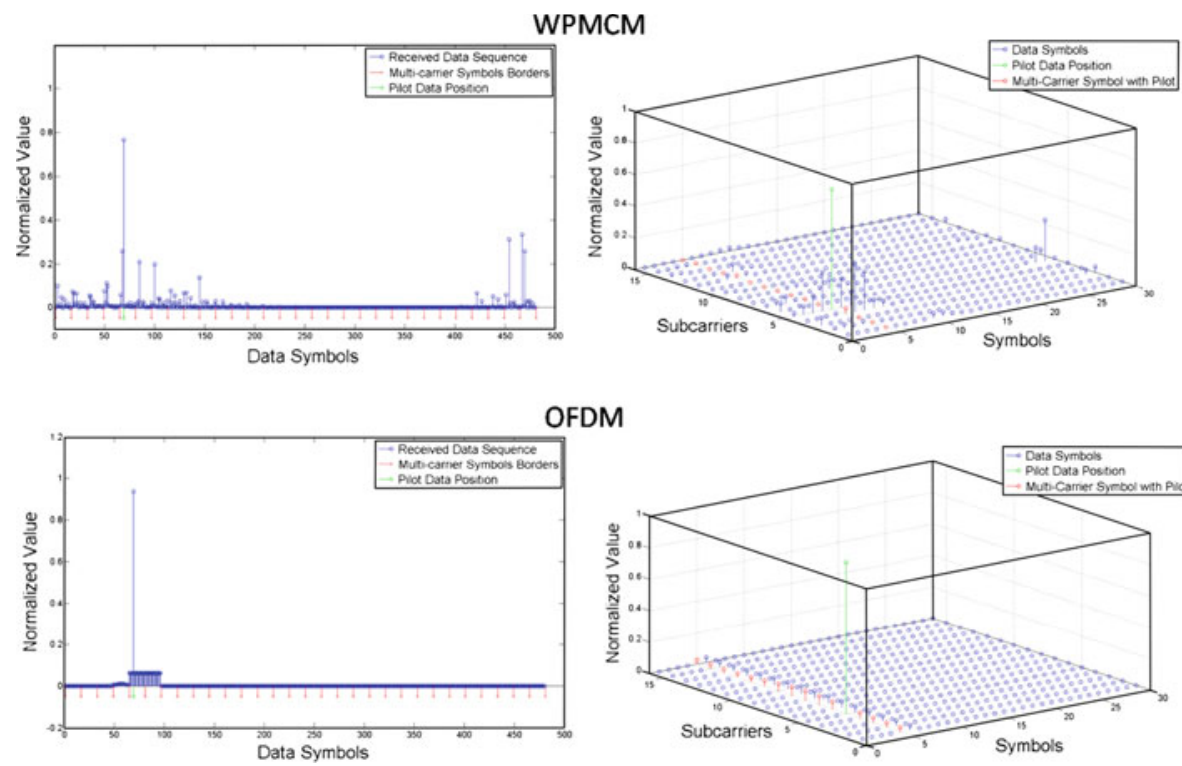

OFDM

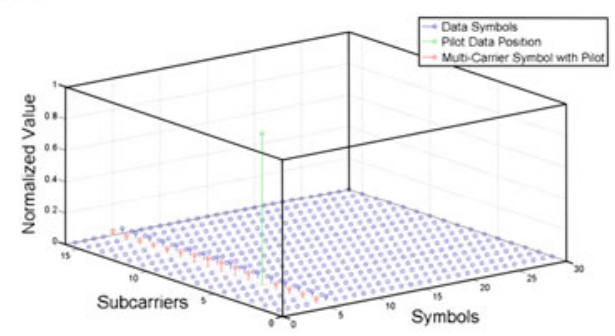

Fig. 17 Received subcarriers spectral energy in a frame in presence of timing error; Up: WPMCM with Daubechies wavelet, Down: OFDM

pilot symbol is spread into the subsequent symbol (ISI) but not into the previous symbol. Furthermore, energy of pilot subcarrier is also spread across the other subcarriers located in the same symbol (ICI).

In case of WPMCM the energy of single pilot is spread across a number of symbols, where subcarriers close to the pilot subcarrier contain the greatest part of interfering energy, regardless of the symbol index.

\section{Conclusion}

WPMCM is relatively a young and promising communication concept. It shares most of the characteristics of an orthogonal multi carrier system and in addition offers the advantage of flexibility and adaptation. These properties can make it suitable for the design and development of communication systems for the future (Cognitive Radio and 4G). However, to realize this vision there are potential pitfalls that have to be ironed out. In this paper we presented one of the major drawbacks of WPMCM in comparison with classical OFDM. OFDM uses the Cyclic Prefix (CP) or Guard Intervals (GI) to improve tolerance to timing error and/or dispersive channel. WPMCM, as a consequence of the time overlap nature of its symbols cannot use CP or GI. Furthermore, the ISI in OFDM is normally generated due to overlap of two consecutive symbols. However in the case of WPMCM the ISI is generated due to a number of adjacent symbols overlapping over-one-another. Because of this, WPMCM is very sensitive to even small timing discrepancies between transmitter and receiver, as can be seen from simulation results shown in Sect.5. During simulations several known wavelets including the popular Daubechies family and its variants Symlets, Coiflets as well as the discrete Meyer and biorthogonal wavelets were applied and studied. All of these families were found to be highly sensitive to time offset. When the characteristics, such as length of filter, amongst the members of the same family were altered there were no perceivable differences in the system performance. 
It is worth mentioning here that the performances of WPMCM matches well with that of OFDM with regard to phase and frequency offset errors [37]. Furthermore, the Peakto-Average Power Ratio (PAPR) performance of WPMCM and OFDM are comparable [38].

\section{Further Work}

The wavelets used in this article are standard wavelets that were developed for applications such as image processing or encryption, and hence may not be entirely suitable for the MCM. Future work should consider design of wavelet and scaling filters that would minimize the interference energy which arises as a result of timing error [31,32]. Another possible solution is to use complex wavelets to reduce WPMCM time shift sensitivity [33]. Furthermore, a robust synchronization scheme that can tackle relatively large timing offsets can be developed and applied [34-36].

Open Access This article is distributed under the terms of the Creative Commons Attribution Noncommercial License which permits any noncommercial use, distribution, and reproduction in any medium, provided the original author(s) and source are credited.

\section{References}

1. Wornell, G. (1996). Emerging applications of multirate signal processing and wavelets in digital communications. Proceedings of IEEE, 84, 586-603.

2. Lakshmanan, M. K., \& Nikookar, H. (2006). A review of wavelets for digital wireless communication. Springer Journal on Wireless Personal Communication, 37(3-4), 387-420.

3. Lindsey, A. (1997). Wavelet packet modulation for orthogonally transmultiplexed communications. IEEE Transaction On Signal Processing, 45, 1336-1339.

4. Pollet, T., van Bladel, M., \& Moeneclaey, M. (1995). BER sensitivity of OFDM systems to carrier frequency offset and wiener phase noise. IEEE Transaction on Communications, 43, 191-193.

5. Armada, A. G. (2001). Understanding the effect of phase noise in OFDM. IEEE Transaction on Broadcasting, 47(2), 153-159.

6. Nikookar, H., \& Prasad, R. (1996). On the sensitivity of multicarrier transmission over multipath channels to phase noise and frequency offset. In Proceedings on 7th IEEE international symposium personal, indoor mobile radio communication (PIMRC '96), Vol. 1, (pp. 68-72), October 1996.

7. Schenk, T. C. W., van der Hofstad, R. W., \& Fledderus, E. R. (2007). Distribution of the ICI term in phase noise impaired OFDM systems. IEEE Transaction on Wireless Communications, 6(4), 1488-1500.

8. Steendam, H., \& Moeneclaey, M. (2000). Sensitivity of orthogonal frequency division multiplexed systems to carrier and clock synchronization errors. Elsevier Signal Processing, 80(7), 1217-1229.

9. Tomba, L. (1998). On the effect of Wiener phase noise in OFDM systems. IEEE Transaction on Communications, 46(5), 580-583.

10. Sathananthan, K., \& Tellambura, C. (2001). Probability of error calculation of OFDM system with frequency offset. IEEE Transaction on Communications, 49(11), 1884-1888.

11. Nikookar, H., \& Negash, B. G. (2000). Frequency offset sensitivity reduction of multicarrier transmission by waveshaping. In Proceedings of IEEE international conference on personal wireless communications (ICPWC '2000) (pp. 444-448)

12. Armstrong, J. (1999). Analysis of new and existing methods of reducing intercarrier interference due to frequency offset in OFDM. IEEE Transaction on Communications, 47(3), 365-369.

13. Moose, P. H. (1994). A technique for orthogonal frequency division multiplexing frequency offset correction. IEEE Transaction on Communications, 42(10), 2908-2914.

14. Armada, A. G., \& Calvo, M. (1998). Phase noise and sub-carrier spacing effects on the performance of an OFDM communication system. IEEE Communication Letters, 2(1), 11-13.

15. Lion, Y. C., \& Chen, K. C. (2005). Estimation of Wiener phase noise by the autocorrelation of the ICI weighting function in OFDM system. In Proceedings of 16th IEEE international symposium on personal, indoor and mobile radio communications (PIMRC '05) (pp. 725-729) 
16. Sandell, M., Beekm, J. J., \& Brjesson, P. O. (1995). Timing and frequency synchronization in OFDM systems using the cyclic prefix. In International symposium on synchronization (pp. 16-19)

17. Tanda, M. (2004). Blind symbol timing and frequency offset estimation in OFDM systems with real data symbols. IEEE Transactions on Communications, 52(10), 1609-1612.

18. Athaudage, C. R. N. (2002). BER sensitivity of OFDM systems to time synchronization error. In Proceedings of IEEE international conference on communication systems (ICCS 2002), Vol. 1, pp. 42-46.

19. Mostofi, Y., \& Cox, D. C. (2002). Mathematical analysis of the impact of timing synchronization errors on the performance of an OFDM system. IEEE Transaction on Communications, 54(2), 226-230.

20. Liu, D., Tang, Y., Sang, D., \& Li, S. (2004). Impact of the timing error on BER performance of TDD pre-equalized OFDM system. In Proceedings of IEEE international conference on personal wireless communications (ICPWC '2000), Vol. 1 (pp. 714-718)

21. Lee, D., \& Cheun, V. (2002). Coarse symbol synchronization algorithms for OFDM systems in multipath channels. IEEE Communication Letters, 6(10), 446-448.

22. Coulson, A. J. (2001). Maximum likelihood synchronization for OFDM using a pilot symbol algorithms. IEEE Journal on Selected Areas in Communications, 19(12), 2486-2494.

23. Yang, B., Letaief, K. B., Cheng, R. S., \& Cao, Z. (2000). Timing recovery for OFDM transmission. IEEE Journal on Selected Areas in Communications, 18(11), 2278-2291.

24. Khoirul, A., Priantoro, A.U., Saito, M., Hara, T., Okada, M., \& Yamamoto, H. (2004). On the PAPR reduction for wavelet based transmultiplexer. International symposium on communications and information technologies (pp. 812-815), October 2004.

25. Lakshmanan, M. K., Budiarjo, I., \& Nikookar, H. (2007). Maximally frequency selective wavelet packets based multi-carrier modulation scheme for cognitive radio systems. In Proceedings of 50th global communications conference (GLOBECOM), Washington DC, USA.

26. Gautier, M., Lereau, C., Arndt, M., \& Lienard, J. (2008). PAPR analysis for wavelet packet modulation. IEEE international symposium on communications (ISCCSP '08).

27. Slimane, S. B. (2006). Peak-to-average power ratio reduction of OFDM signals using pulse shaping. IEEE global telecommunications conference, Vol. 41 (pp. 1412-1416)

28. Lakshmanan, M. K., \& Nikookar, H. (2007). Wavelet packet based strategy to mitigate wideband interference on impulse radio. IEEE (PIMRC '2007) Personal, indoor and mobile radio communications, (pp. 1-5)

29. Vaidyanathan, P. P. (1993). Multirate systems and filter banks. Upper Saddle River, New Jersey: Prentice-Hall Inc.

30. Burrus, C. S., Gopinath, R. A., \& Guo, H. (1998). Introduction to wavelets and wavelet transforms, a primer. Upper Saddle River, NJ, USA: Prentice Hall.

31. Wu, J. (1997). Wavelet packet division multiplexing. Ph.D. Dissertation, Department of Electrical and Computer Engineering, McMaster University, Hamilton, Ontario, Canada

32. Wong, K. M., Wu, J., Davidson, T. N., \& Jin, Q. (1997). Packet division multiplexing and wavelet packet design under timing error effects. IEEE Transactions on Signal Processing, 45, 2877-2890.

33. Gautier, M., \& Lienard, J. (2006). Performances of complex wavelet packet based multicarrier transmission through dispersive channel. IEEE Nordic Signal Processing Symposium (NORSIG '06), Iceland.

34. Jamin, A., \& Mähönen, P. (2006). Mueller and Muller algorithm based synchronisation for wavelet packet modulation. In Proceedings of ACM IWCMC (International Wireless Communications and Mobile Compo Conference), Vancouver, Canada.

35. Luise, M., Marselli, M., \& Reggiannini, R. (2000). Synchronization for wavelet-based multirate transmissions. IEEE Transactions on Communications, 48(6), 1047-1054.

36. Lallart, M., Nolan, K. E., Sutton, P., \& Doyle, L. E. (2007). On-The-Fly synchronization using wavelet and wavelet packet OFDM. In Proceedings of 13th European Wireless Conference, France.

37. Karamehmedović, D., Lakshmanan, M. K., \& Nikookar, H. (2009). Performance of WPMCM and OFDM in the presence of carrier frequency offset and phase noise. Journal of Communications (JCM), 4(7), 496-508.

38. Torun, B., Lakshmanan, M. K., \& Nikookar, H. (2009). Peak-to-average power ratio studies for wavelet packet modulation. European Wireless Technology Conference. Rome, Italy. 


\section{Author Biographies}

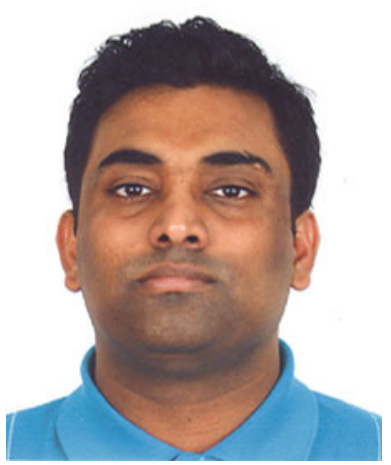

M. K. Lakshmanan received the B.E. (with distinction) in electrical engineering from the University of Madras, Chennai, India, in 2000. In 2000, he joined the Indian Software firm, Polaris Software Labs Ltd., where he wrote software for Telecommunication applications. At Polaris, he was awarded the "On The Spot Of Excellence Award" for his efforts. In 2003, he moved to the Indian Institute of Technology-Madras, India, to lead a team of junior researchers to develop and establish a wireless communications network for rural connectivity. In 2004, he was granted the Royal Dutch/Shell Chevning scholarship to pursue a Masters program in Telecommunications at the Delft University of Technology (TU Delft). He completed his Masters (with CUM LAUDE) in the 2006 and continued with his Ph.D. studies at TU Delft where he is currently conducting research in the fields of wavelets, signal processing and Wireless Communications at the International Research Center for Telecommunications Transmission and Radar (IRCTR). In December 2007 he won the BEST STUDENT PAPER award at the 10th International Symposium on Wireless Personal Multimedia Communications, Jaipur, India.

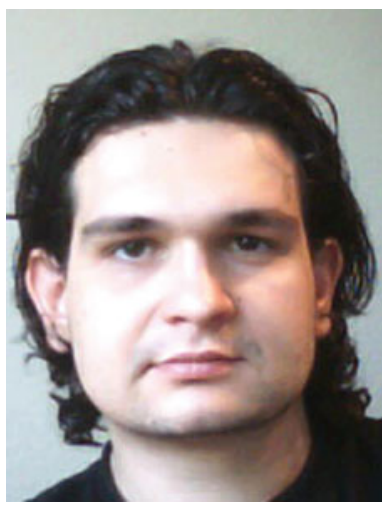

D. Karamehmedović was born in Sarajevo, Bosnia and Herzegovina in 1980. He received both the B.Sc. and M.Sc. degrees in Electrical Engineering from the Delft University of Technology (TU Delft), The Netherlands, in 2004 and 2009 respectively. He is founder of company GetNET where he held manager function from 2003 till 2007. In 2009 he joined Frames Process Systems where he is working on instrumentation design and development.

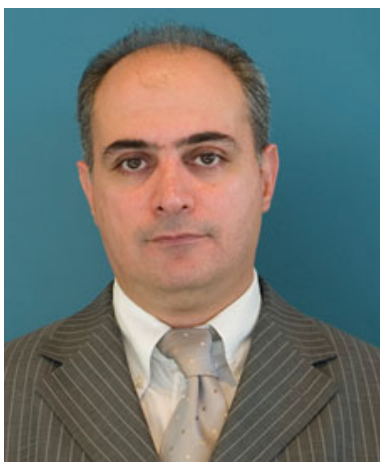

H. Nikookar received his Ph.D. in Electrical Engineering from Delft University of Technology (TU Delft), The Netherlands, in 1995. He is an Associate Professor at the International research Centre for Telecommunications and Radar (IRCTR) of the Department of Electrical Engineering, Mathematics and Computer Science of TU Delft. He is also the leader of the Radio Advanced Technologies and Systems (RATS) program of the IRCTR. Dr. Nikookar has conducted research in many areas of wireless communications, including wireless channel modeling, UWB, MIMO, multicarrier transmission, Wavelet-based OFDM and Cognitive Radio. He is a senior member of the IEEE and the coauthor of the Book, Introduction to Ultra Wideband for Wireless Communications, Springer, 2008. 